\title{
Considering the Impact of Large-Scale Network Interactions on Cognitive Control
}

\author{
(1)Fiona Kumfor, ${ }^{1,2,3}$ Nadene Dermody, ${ }^{1,4}$ and Muireann Irish ${ }^{1,2,4}$ \\ ${ }^{1}$ Neuroscience Research Australia, and ${ }^{2}$ ARC Centre of Excellence in Cognition and its Disorders, ${ }^{3}$ School of Medical Sciences, and ${ }^{4}$ School of Psychology, \\ University of New South Wales, Sydney, NSW 2031, Australia \\ Review of Jilka et al.
}

In everyday life, we often face situations in which we must compete with an overriding/pre-potent response to inhibit our behavior and act in line with situational demands. For example, on most days, the drive home from work is a relatively automatic behavior that can be undertaken with limited conscious effort. But if a dog ran unexpectedly into traffic, you would need to quickly identify this change in the environment and stop your ongoing habitual actions to prevent an accident. This ability to rapidly halt actions that are already underway in response to a change in the environment or internal state is dependent on cognitive control; the ability to "override or augment reflexive behavior and habitual reactions to orchestrate behavior in accord with our intentions" (Miller, 2000, p. 59).

The advent of resting-state functional connectivity neuroimaging techniques has led to the delineation of several largescale functional brain networks that are differentially recruited contingent on situational demands. Two key networks are the salience network and the default mode network (DMN). The salience network

Received Oct. 9, 2014; revised Nov. 12, 2014; accepted Nov. 14, 2014.

M.I. is supported by an ARC Discovery Early Career Researcher Award (DE130100463).

Correspondence should be addressed to Dr. Muireann Irish, Neuroscience Research Australia, Sydney, NSW 2031, Australia. E-mail: m.irish@neura.edu.au.

DOI:10.1523/JNEUROSCI.4213-14.2015

Copyright $\odot 2015$ the authors $\quad 0270-6474 / 15 / 350001-03 \$ 15.00 / 0$ is recruited in response to attentiongrabbing changes in the environment, and it is anchored by the dorsal anterior cingulate cortex and orbital frontoinsular cortices, with robust connections with subcortical and limbic structures (Zhou et al., 2010). Conversely, the DMN is activated when current situational demands are insufficient to capture our attention (e.g., during monotonous tasks); it encompasses a distributed set of regions including the medial and lateral temporal cortices and inferior lateral parietal cortices, centered on midline "hubs," including the dorsomedial prefrontal and posterior cingulate cortices (Buckner et al., 2008). Crucially, the communication within, and the interaction between, large-scale networks is necessary for adaptive cognitive function. Cognitive control therefore provides an interesting conceptual model for investigating the potential interactions between large-scale brain networks proposed to support higher-order cognitive processes.

Cognitive control is often compromised after traumatic brain injury (TBI), with individuals experiencing severe difficulties in inhibiting and regulating their behavior to meet internal goals. Diffuse axonal injury occurs after TBI, and the integrity of white matter pathways connecting network hubs is disrupted. Thus, large-scale network function is impaired (Sharp et al., 2014). Studying individuals with TBI can therefore provide important insights into the contribution of large-scale network dysfunction on cognition and behavior. To this end, a recent study by Jilka et al. (2014) used behavioral and neuroimaging methods to determine how cognitive control, and the underlying neural networks subtending this process, is altered by TBI.

Previous work by the authors revealed that TBI-related damage to the white matter tract connecting the right anterior insula to the presupplementary motor area/dorsal anterior cingulate cortex (rAI-preSMA/dACC), located within the salience network, is associated with a failure to deactivate the DMN (Bonnelle et al., 2012). In their recent study, Jilka et al. (2014) sought to extend this research to determine whether damage to the rAI-preSMA/dACC tract impairs dynamic interactions between the salience network and the DMN, manifesting in disrupted cognitive control after TBI.

The authors used two tasks to investigate the capacity for cognitive control in TBI relative to healthy control participants: a stop signal task and a motor switching task. On the stop signal task, participants were shown either left or right arrows, and were asked to press the corresponding left or right key. On a minority of trials (20\%), however, participants were shown a "stop signal" (red dot) and were required to inhibit their response. In contrast, on the motor switching task, participants learned to respond 
to blue targets with their left hand and red targets with their right hand. Crucially, on a minority of trials (20\%), participants were instructed to switch their response. The authors used psychophysiological interaction analysis (PPI) of functional MRI data collected as subjects performed these two tasks to investigate functional connectivity between salience network regions of interest (i.e., right anterior insula, dorsal anterior cingulate cortex) and the DMN, as well as a region of interest outside the salience network (i.e., right inferior frontal gyrus) and the DMN.

The results revealed that in controls, successful performance on both the inhibition and switching tasks was associated with stronger correlations (increased functional connectivity) between the right anterior insula node of the salience network and the DMN. Importantly, deficits in inhibition and switching seen behaviorally in TBI were associated with decreased functional connectivity between the right anterior insula node and the DMN compared with controls. Moreover, in TBI, greater damage to the rAI-preSMA/dACC tract within the salience network was associated with weaker functional connectivity between the right anterior insula and the DMN during inhibition and switching. Together, these results indicate that the right anterior insula represents a crucial node within the salience network, which potentially enables dynamic interactions between the salience and default mode networks.

The study by Jilka et al. (2014) provides an elegant demonstration of the dynamic interactions between functional brain networks in supporting complex cognitive acts essential for adaptive functioning. The exposition of specific sites within the salience network (i.e., the right anterior insula) that potentially support interactions between the salience network and the DMN is noteworthy and corroborates previous work implicating the right anterior insula and anterior cingulate cortex across a range of higher-order processes including cognitive control and performance monitoring. It has been suggested that these regions form a highly interconnected core system for taskdependent control of goal-directed behavior and sensory processing (Dosenbach et al., 2007). One remaining issue, however, concerns how the current findings relate to influential theories which view the rostral prefrontal cortex as a gateway between monitoring of external states and the flexible switching of attention to selfgenerated, internal states (Burgess et al., 2007). A further region of interest in this regard is the posterior cingulate cortex, one of the putative midline hubs of the DMN, as this region has been proposed to interact with frontoparietal attentional networks to regulate between internal and external forms of cognition (Leech et al., 2012).

We suggest that exploration of the functional coupling between the salience network and other functional brain networks via the insula might further our understanding of cognitive control from a network perspective. While Jilka et al. (2014) interpreted their findings in terms of the functional coupling between the salience network and the DMN, the right frontoinsular node of the salience network has also been implicated in switching between the DMN and the central-executive network (Sridharan et al., 2008). This large-scale functional brain network encompasses the dorsolateral prefrontal cortex and the posterior parietal cortex and is important for higher-level goaldirected behavior, decision-making, and working memory. The insula is uniquely positioned to serve as an interface between the salience network, DMN, and central-executive network, with reciprocal connections between sensory, motor, limbic, and association brain regions, enabling integration of information from across the brain. How the findings by Jilka et al. (2014) fit within this broader framework of largescale network interactions will be an important area for future research.

A second important consideration is how such network interactions may be affected in other clinical populations. Similar to TBI, neurodegenerative disorders are increasingly being viewed as "network phenomena" and thus offer a unique opportunity to study the impact of largescale functional network disruption on complex behaviors. The behavioral variant of frontotemporal dementia represents a syndrome of immense interest in this context, given that the earliest sites of pathology reside in the frontoinsular cortices, reflecting progressive degeneration of the salience network (Zhou et al., 2010). Clinically, these patients display disinhibited and inappropriate behaviors, pointing toward impaired cognitive control, which is generally taken to reflect prefrontal cortical degeneration. Recent work, however, has revealed that impairments in higher-level cognitive abilities in frontotemporal dementia, such as moral reasoning, are associated with abnormal modulation of the DMN by the salience network (Chiong et al., 2013). This finding, together with Jilka et al.'s (2014) results, has important implications for other cognitive and behavioral features of this syndrome. For example, socially disinhibited behavior is a prominent feature of frontotemporal dementia. Given these new insights into the putative role of the salience network, it is reasonable to propose that in social settings the salience network must first identify the presence of relevant social cues, following which activation of the central-executive network is coordinated to guide decision-making regarding appropriate behavior. In this way, degeneration of the insula node of the salience network may prevent the flexible switching between the centralexecutive network and the DMN in response to contextual/environmental demands, potentially giving rise to a number of hallmark behavioral and cognitive abnormalities observed in this syndrome. While our interpretation at this point is purely speculative, examination of the characteristic impairments in frontotemporal dementia, from this network perspective, via targeted tasks assessing cognitive control, will provide important insights into the role of largescale neural network interactions in supporting cognition and behavior.

In conclusion, Jilka et al.'s (2014) study localizes the right anterior insula as a key node within the salience network for modulating large-scale functional brain network activity to support cognitive control. We propose that future work adopting this convergent approach will prove particularly useful for understanding the origins of a host of dysfunctional behaviors typically observed in neurodegenerative and psychiatric populations.

\section{References}

Bonnelle V, Ham TE, Leech R, Kinnunen KM, Mehta MA, Greenwood RJ, Sharp DJ (2012) Salience network integrity predicts default mode network function after traumatic brain injury. Proc Natl Acad Sci U S A 109:46904695. CrossRef Medline

Buckner RL, Andrews-Hanna JR, Schacter DL (2008) The brain's default network. Ann N Y Acad Sci 1124:1-38. CrossRef Medline

Burgess PW, Dumontheil I, Gilbert SJ (2007) The gateway hypothesis of rostral prefrontal cortex (area 10) function. Trends Cogn Sci 11: 290-298. CrossRef Medline

Chiong W, Wilson SM, D’Esposito M, Kayser AS, Grossman SN, Poorzand P, Seeley WW, Miller BL, Rankin KP (2013) The salience network causally influences default mode 
network activity during moral reasoning. Brain 136:1929-1941. CrossRef Medline

Dosenbach NU, Fair DA, Miezin FM, Cohen AL, Wenger KK, Dosenbach RA, Fox MD, Snyder AZ, Vincent JL, Raichle ME, Schlaggar BL, Petersen SE (2007) Distinct brain networks for adaptive and stable task control in humans. Proc Natl Acad Sci U S A 104:11073-11078. CrossRef Medline

Jilka SR, Scott G, Ham T, Pickering A, Bonnelle V, Braga RM, Leech R, Sharp DJ (2014) Damage to the salience network and interactions with the default mode network. J Neurosci 34: 10798-10807. CrossRef Medline

Leech R, Braga R, Sharp DJ (2012) Echoes of the brain within the posterior cingulate cortex. J Neurosci 32:215-222. CrossRef Medline

Miller EK (2000) The prefontral cortex and cognitive control. Nat Rev Neurosci 1:59-65. CrossRef Medline

Sharp DJ, Scott G, Leech R (2014) Network dysfunction after traumatic brain injury. Nat Rev Neurol 10:156-166. CrossRef Medline
Sridharan D, Levitin DJ, Menon V (2008) A critical role for the right fronto-insular cortex in switching between central-executive and default-mode networks. Proc Natl Acad Sci U S A 105:12569-12574. CrossRef Medline

Zhou J, Greicius MD, Gennatas ED, Growdon ME, Jang JY, Rabinovici GD, Kramer JH, Weiner M, Miller BL, Seeley WW (2010) Divergent network connectivity changes in behavioural variant frontotemporal dementia and Alzheimer's disease. Brain 133:13521367. CrossRef Medline 